\title{
Nationwide survey of Baller-Gerold syndrome in Japanese population
}

\author{
HIDEO KANEKO $^{1}$, RIE IZUMI $^{2}$, HIROTSUGU ODA $^{3}$, OSAMU OHARA $^{4}$, \\ KIYOKO SAMESHIMA ${ }^{5}$, HIDENORI OHNISHI ${ }^{6}$, TOSHIYUKI FUKAO ${ }^{6}$ and MICHINORI FUNATO ${ }^{1}$ \\ ${ }^{1}$ Department of Clinical Research, National Hospital Organization Nagara Medical Center, \\ Gifu 502-8558; ${ }^{2}$ Niigata Prefecture Hamagumi Medical Rehabilitation Center for Children, Niigata 951-8121; \\ ${ }^{3}$ Laboratory for Integrative Genomics, RIKEN Center for Integrative Medical Sciences (RIKEN-IMS), Yokohama, \\ Kanagawa 230-0045; ${ }^{4}$ Department of Technology Development, Kazusa DNA Research Institute, Kisarazu, \\ Chiba 292-0818; ${ }^{5}$ Division of Medical Genetics, Gunma Children's Medical Center, Shibukawa, Gunma 377-8577; \\ ${ }^{6}$ Department of Pediatrics, Graduate School of Medicine, Gifu University, Gifu 501-1194, Japan
}

Received July 19, 2016; Accepted March 10, 2017

DOI: $10.3892 / \mathrm{mmr} .2017 .6408$

\begin{abstract}
Baller-Gerold syndrome (BGS) is a rare autosomal genetic disorder characterized by radial aplasia/hypoplasia and craniosynostosis. The causative gene for BGS encodes RECQL4, which belongs to the RecQ helicase family. To understand BGS patients in Japan, a nationwide survey was conducted, which identified 2 families and 3 patients affected by the syndrome. All the three patients showed radial defects and craniosynostosis. In one patient who showed a dislocated joint of the hip and flexion contracture of both the elbow joints and wrists at birth, a homozygous large deletion in the RECQL4 gene was identified. This is the first reported case of BGS in Japan caused by RECQL4 gene mutation.
\end{abstract}

\section{Introduction}

There are five human RecQ-like proteins (RECQL1, BLM, WRN, RECQL4 and RECQ5), each having 3' to 5' DNA helicase activity but little sequence similarity outside the helicase motifs (1). Three of these helicases encode causative genes for Bloom syndrome (BLM), Werner syndrome (WRN) and Rothmund-Thomson syndrome (RECQL4), respectively. These three syndromes show genomic instability and cancer susceptibility, but each also has distinctive features (2). RECQL4 is the causative gene for Rothmund-Thomson syndrome (OMIM 266280) characterized by poikiloderma and skeletal defects. Homozyogous mutations or compound heterozygous

Correspondence to: Professor Hideo Kaneko, Department of Clinical Research, National Hospital Organization Nagara Medical Center, 1300-7 Nagara, Gifu 502-8558, Japan

E-mail: hkaneko@nagara-lan.hosp.go.jp

Key words: Baller-Gerold, RECQL4 gene, large deletion mutations of the RECQL4 gene causes Rothmund-Thomson syndrome $(3,4)$.

However, mutations in the RECQL4 gene have been associated with two other recessive disorders: One is RAPADILINO syndrome (OMIM 266280) which is characterized by radial hypoplasia, patella hypoplasia and arched plate, diarrhoea and dislocated joints, little size and limb malformation, slender nose and normal intelligence (4). The other is Baller-Gerold syndrome (BGS) (OMIM 218600) characterized by radial aplasia/hypoplasia and craniosynostosis (5). Three syndromes have overlapping features, such as short stature and radial ray abnormalities (6).

In Japan there have been no reports on BGS. We have performed a nationwide questionnaire based study of BGS. Three BGS patients were identified: one was the sporadic case with BGS, and the remaining two were brothers. In this patient, we found for the first time a homozygous large deletion in the RECQL4 gene in Japan.

\section{Materials and methods}

Patients and questionnaire. From 2012 to 2014, a preliminary questionnaire for soliciting information about BGS patients was sent to 1,407 Departments of 515 Pediatrics, 515 Dermatology Departments and 377 Cancer Hospitals in Japan. The response rate was 83, 68 and 36\%, respectively. Parents of the three patients suspected of having GBS were requested to fill out more extensive questionnaire to obtain detailed information on the three patients. The analyses were approved by the ethics committee of Nagara Medical Center, and written informed consent was obtained from the patients and/or their parents.

Genetic analysis. Peripheral blood monocytes cells (PBMCs) were separated using Ficoll-Paque (Amersham Bioscience, Uppsala, Sweden). Genomic DNA from PBMCs of the patient and his parents were prepared using a Sepa Gene kit (Sankyo Jyunyaku, Tokyo, Japan). Amplification of the full-length RECQL4 gene region was performed using PrimeSTAR 

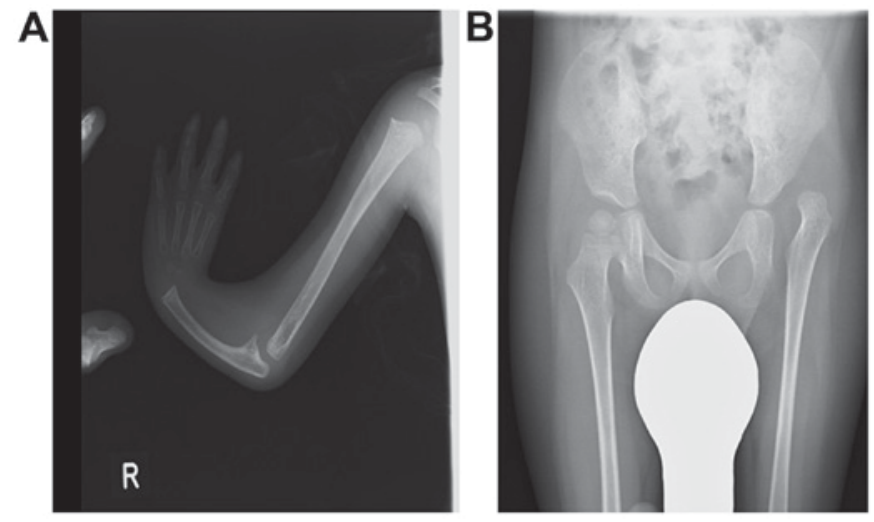

Figure 1. Skeletal anomalies of case 3 revealed by X-ray examination. Right radial ray defects; (A) absent radius and absent thumb. (B) Left hip dislocation.

GXL DNA polymerase (Takara Bio, Inc., Shiga, Japan), using primers 5'-ATTGGCTGCTTGTCCGAG-3' and 5'-GCCTGG AATATGTGATGTGC-3'. The PCR products were electrophoresed on $0.7 \%$ agarose gel, and were also sequenced using Big Dye Terminator v3.1 (Thermo Fisher Scientific, Waltham, MA, USA), using primers 5'-GGTGAGCCATATGTGAAC TGG-3' and 5'-CACTGCATCCACAGAGCAAG-3'.

\section{Results}

Three patients in two families were identified to be affected by BGS by the questionnaire-based survey in Japan. One family had a 3-year-old older brother (case 1) and a 1-year-old younger brother (case 2) with BGS. The older brother showed craniosynostosis, thumb hypoplasia, radial ray defects and imperforate anus and nasolacrimal duct malposition. Operations were performed for craniosynostosis, imperforate anus and nasolacrimal duct malposition. The younger brother showed left ptosis and was diagnosed as having BGS on the basis of his clinical features and the finding of the X-ray examination of the systemic bone. Their RECQL4 genes were not analyzed because informed consents were not obtained.

Case 3 was a 4-year-old boy. In addition to craniosynostosis and thumb hypoplasia (Fig. 1), he showed left hip dislocation, left knee joint dislocation, bent elbows, and excessive hand abduction. His intelligence is within the normal range, as determined by a new edition K-type development inspection. At 4 years of age he was still not ambulatory but could sit.

From his clinical features and bone X-ray examination findings he was suspected of having BGS. Therefore, his RECQL4 gene was analyzed. His RECQL4 gene showed a 1,614 bp homozygous deletion and $1 \mathrm{bp}(\mathrm{G})$ insertion (NC_000008.10:g.145737562_145739175delinsC) (Fig. 2A). Deleted DNA spanned from intron 12 to the former part of exon 18, which contained the helicase motif (Fig. 3). Agarose gel analysis (Fig. 2B) showed that his father and mother carried the wild type and mutant RECQL4 genes, respectively.

\section{Discussion}

The prevalence of BGS is unknown; it is probably less than 1:1,000,000 (7). This rarity of BGS makes its diagnosis

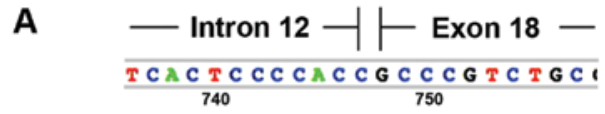

Patient
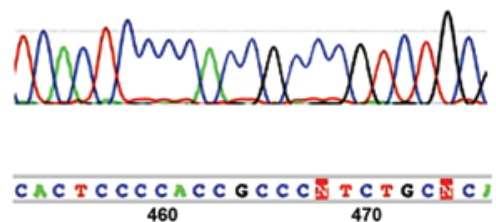

470
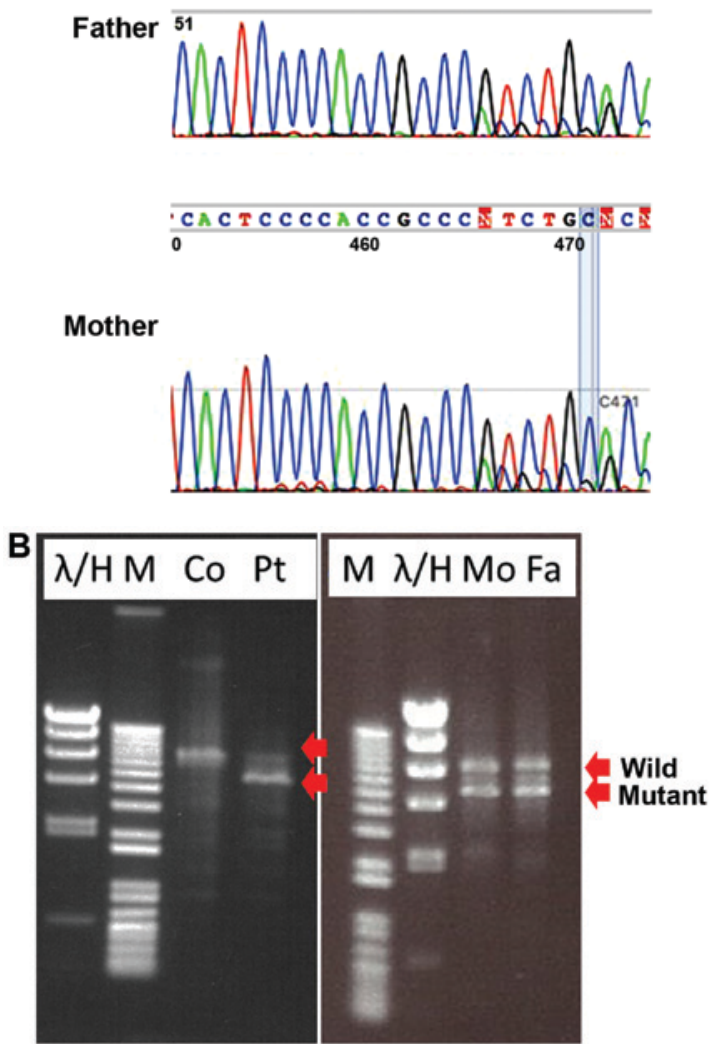

Figure 2. (A) Chromatogram of DNA sequences at DNA break point (NC_000008.10:g.145737562_145739175delinsC). (B) Large homozygous deletion of RECQL4 gene in case 3 detected by agarose gel electrophoresis. $\lambda / \mathrm{H}$, HindIII digested DNA marker, M, DNA marker, Co, control, Pt, case 3 , Mo, Mother, Fa, Father.

\section{CCCCACTGCCCACG CCAGGCCCTGGCCCGTC}

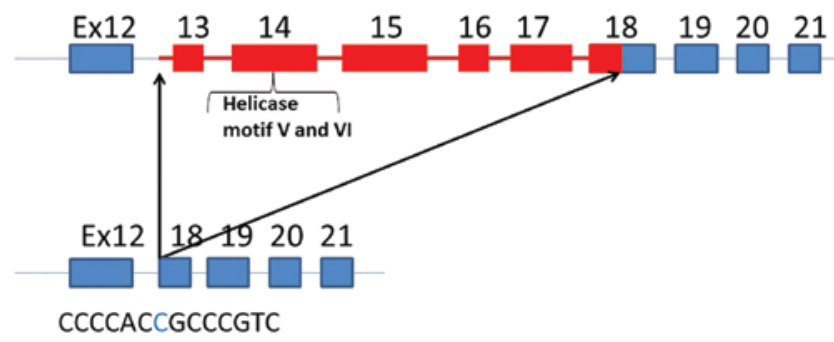

Figure 3. In case 3, detected homozygous deletion of the RECQL4 gene from intron 12 to the former part of exon 18, resulted in the deletion of amino acids after Ala687. Red boxes indicated the deleted exons and introns. Exon 14 encodes the helicase motif V and VI (1).

difficult in some cases. Piard et al (8). Showed that the mean age at referral for BGS was 7 years. In our nationwide survey only two families were identified to have members affected 
by BGS. All of the affected members had craniosynostosis and radial ray defects. On the other hand Rothmund-Thomson syndrome and RAPADILINO syndrome are two recessively inherited syndromes whose clinical features overlap those of BGS. Rothmund-Thomson syndrome is characterized by poikiloderma. Radial ray hypoplasia or absent thumbs occur in a minority of cases. RAPADILINO syndrome is characterized by radial ray defects. BGS is characterized by craniosynostosis in association with radial hypoplasia. Our cases showed radial ray defect with carniosyonostosis without poikiloderma which is consistent with the characteristic of BGS.

The RECQL4 gene in our genetically analyzed patient showed homozygous deletion from intron 12 to the former part of exon 18 resulting in the deletion of amino acids after Ala687. To our knowledge this large deletion of RECQL4 gene has not been reported in other BGS patients. This deletion spans the RecQ helicase motif from IV to VI. The deleted RECQL4 protein in the patient might not be functional. To the best of our knowledge, this deletion in the RECQL4 gene has not been reported. Piard et al reported that no RECQL4 mutations were found in their BGS group without poikiloderma. However, our patient had café-au-lait-like spots but not poikiloderma (8). The relationship between poikiloderma and RECQL4 gene mutation should be further examined.

In our patient the parents had heterozygous RECQL4 gene mutation without evident consanguinity. A molecular study-based diagnosis is powerful tool for genetic counselling of individuals affected by BGS. Cao et al Reported the case of BGS prenatally diagnosed (9). The cases of BGS diagnosed on the basis of molecular genetics should be accumulated.

\section{Acknowledgements}

This study was supported in part by Health and Labor Science Research Grants for Research on Intractable Diseases from The Ministry of Health, Labour and Welfare of Japan (H23-075).

\section{References}

1. Nakayama K: RecQ family helicases: Roles as tumor suppressor proteins. Oncogene 21: 9008-9021, 2002.

2. Kaneko $\mathrm{H}$ and Kondo N: Clinical features of Bloom syndrome and function of the causative gene, BLM helicase. Expert Rev Mol Diagn 4: 393-401, 2004.

3. Kitao S, Shimamoto A, Goto M, Miller RW, Smithson WA, Lindor NM and Furuichi Y: Mutations in RECQL4 cause a subset of cases of Rothmund-Thomson syndrome. Nat Genet 22: 82-84, 1999.

4. Larizza L, Roversi G and Volpi L: Rothmund-Thomson syndrome. Orphanet J Rare Dis 5: 2, 2010.

5. Van Maldergem L, Verloes A, Lejeune L and Gillerot Y: The Baller-Gerold syndrome. J Med Genet 29: 266-268, 1992.

6. Van Maldergem L, Siitonen HA, Jalkh N, Chouery E, De Roy M, Delague V, Muenke M, Jabs EW, Cai J, Wang LL, et al: Revisiting the craniosynostosis-radial ray hypoplasia association: Baller-Gerold syndrome caused by mutations in the RECQL4 gene. J Med Genet 43: 148-152, 2006.

7. Van Maldergem L. In: Pagon RA, Adam MP, Ardinger HH, Wallace SE, Amemiya A, Bean LJH, Bird TD, Fong CT, Mefford HC, Smith RJH and Stephens K (eds). Baller-Gerold Syndrome. Seattle (WA), University of Washington, Seattle, 1993-2016, 2007.

8. Piard J, Aral B, Vabres P, Holder-Espinasse M, Mégarbané A, Gauthier S, Capra V, Pierquin G, Callier P, Baumann C, et al: Search for RECQL4 mutations in 39 patients genotyped for suspected Rothmund-Thomson/Baller-Gerold syndromes. Clin Genet 87: 244-251, 2015.

9. Cao DH, Mu K, Liu DN, Sun JL, Bai XZ, Zhang N, Qiu GB and Ma XW: Identification of novel compound heterozygous RECQL4 mutations and prenatal diagnosis of Baller-Gerold syndrome: A case report. Genet Mol Res 14: 4757-4766, 2015. 